\title{
Decision Support System for the Intelligient Identification of Alzheimer using Neuro Fuzzy logic
}

\author{
Obi J.C ${ }^{1}$ and Imainvan A.A ${ }^{2}$. \\ Department of Computer Science, University of Benin, Benin City. Nigeria ${ }^{1 \& 2}$. \\ tripplejo2k2@yahoo. $\mathrm{com}^{1}(+234(0) 8093088218)$ \\ tonyvanni@yahoo. $\mathrm{com}^{2}(+234(0) 7069742552)$
}

\begin{abstract}
Alzheimer Disease $(A D)$ is a form of dementia; it is a progressive, degenerative disease. Alzheimer is a brain disease that causes problems with memory, thinking and behavior. It is severe enough to interfere with daily activities. Alzheimer symptoms are characterized by memory loss that affects day-to-day function, difficulty performing familiar tasks, problems with language, disorientation of time and place, poor or decreased judgment, problems with abstract thinking, misplacing things, changes in mood and behavior, changes in personality and loss of initiative. Neuro-Fuzzy Logic explores approximation techniques from neural networks to find the parameter of a fuzzy system. In this paper, the traditional procedure for the medical diagnosis of Alzheimer employed by physician is analyzed using neuro-fuzzy inference procedure. The proposed system is a useful decision support approach for the diagnosis of Alzheimer.
\end{abstract}

\section{KEYWORDS}

Neural Network, Fuzzylogic, Neuro Fuzzy System \& Alzeimer

\section{Introduction}

Alzheimer's disease (AD) was discovered in 1907 by Alois Alzheimer [6], but was not considered a major disease or disorder until the 1970s [6]. AD is a slowly progressive disease of the brain that is characterized by impairment of memory and eventually by disturbances in reasoning, planning, language, and perception. Many scientists believe that Alzheimer's disease results from an increase in the production or accumulation of a specific protein (beta-amyloid protein) in the brain that leads to nerve cell death. The likelihood of having Alzheimer's disease increases substantially after the age of 70 and may affect around $50 \%$ of persons over the age of 85 . Nonetheless, Alzheimer's disease is not a normal part of aging and is not something that 
inevitably happens in later life. For example, many people live to over 100 years of age and never develop Alzheimer's disease [16].

Alzheimer is closely like to genetic risk factors for Alzheimer's disease. Most patients develop Alzheimer's disease after age 70. However, $2 \%-5 \%$ of patients develop the disease in the fourth or fifth decade of life (40s or 50s). At least half of these early onset patients have inherited gene mutations associated with their Alzheimer's disease. Moreover, the child of a patient with early onset Alzheimer's disease who has one of these gene mutations has a 50\% risk of developing Alzheimer's disease [16].

There is also a genetic risk for late onset cases. A relatively common form of a gene located on chromosome 19 is associated with late onset Alzheimer's disease. In the majority of Alzheimer's disease cases, however, no specific genetic risks have yet been identified. Other risk factors for Alzheimer's disease include high blood pressure (hypertension), coronary artery disease, diabetes, and possibly elevated blood cholesterol [16]. Individuals who have completed less than eight years of education also have an increased risk for Alzheimer's disease. These factors increase the risk of Alzheimer's disease, but by no means do they mean that Alzheimer's disease is inevitable in persons with these factors. Alzheimer's is a class of dementia [16]. Dementia is a general term for loss of memory and other mental abilities severe enough to interfere with daily life. It is caused by physical changes in the brain [1, 20 and 32].

\subsection{Dementia and Alzheimer}

Alzheimer's disease is the most common type of dementia, accounting for 60 to 80 percent of cases. Other types of dementia are $[\mathbf{1 , 2 0 ]}$ :

\subsection{Mild cognitive impairment}

(MCI) a condition in which a person has problems with memory, language, or another mental function severe enough to be noticeable to other people and to show up on tests, but not serious enough to interfere with daily life, because the problems do not interfere with daily activities, the person does not meet criteria for being diagnosed with dementia [1, 20].

\subsection{Vascular dementia}

Vascular dementia is widely considered the second most common type of dementia. It develops when impaired blood flow to parts of the brain deprives cells of food and oxygen. The diagnosis may be clearest when symptoms appear soon after a single major stroke blocks a large blood vessel and disrupts the blood supply to a significant portion of the brain. This situation is sometimes called "post-stroke dementia." There is also a form in which a series of very small strokes, or infarcts, block small blood vessels. Individually, these strokes do not cause major symptoms, but over time their combined effect becomes noticeable. This type is referred to as vascular cognitive impairment (VCI) or multi-infarct dementia. Symptoms of vascular dementia 
can vary, depending on the specific brain areas deprived of blood. Impairment may occur in "steps," where there is a fairly sudden, noticeable change in function, rather than the slow, steady decline usually seen in Alzheimer's disease. The person may have a past history of heart attacks. High blood pressure, high cholesterol, hardening of the arteries, diabetes, or other risk factors for heart disease are often present $[\mathbf{1 , 2 0}]$.

\subsection{Mixed dementia is a condition}

Mixed dementia is a condition in which Alzheimer's disease and vascular dementia occur at the same time. Many experts believe mixed dementia occurs more often than was previously realized and that it becomes increasingly common in advanced age. This belief is based on brain autopsies showing up to 45 percent of people with dementia have signs of both Alzheimer's and vascular disease. The concept of mixed dementia is clinically important because the combination of the two diseases may have a greater impact on the brain than either by itself $[\mathbf{1 , 2 0}]$.

\subsection{Dementia with Lewy}

Dementia with Lewy bodies is characterized by abnormal deposits of a protein called alphasynuclein that form inside the brain's nerve cells. These deposits are called "Lewy bodies" after the scientist who first described them. Lewy bodies have been found in several brain disorders, including dementia with Lewy bodies, Parkinson's disease and some cases of Alzheimer's [1, 20].

\subsection{Parkinson's disease}

Parkinson's disease begins by affecting movement, resulting in tremors and shakiness, stiffness, difficulty with walking and muscle control, lack of facial expression and impaired speech. Parkinson's is another disease in which Lewy bodies are found in the brain. Many individuals with Parkinson's develop dementia in later stages of the disease [1, 20].

\subsection{Frontotemporal dementia}

Frontotemporal dementia is a rare disorder that affects the front (frontal lobes) and the sides (temporal lobes) of the brain. Because these regions often, but not always, shrink, brain imaging can be useful in diagnosis. There is no specific abnormality associated with all cases of frontotemporal dementia. In one type called Pick's disease, there are abnormal microscopic deposits called Pick bodies, but these are not always present $[\mathbf{1 , 2 0 ] .}$

\subsection{Creutzfeldt-Jakob disease (CJD)}

Creutzfeld-Jacob disease (pronounced CROYZ-felt $Y A H$-cob) is a rare, rapidly fatal disorder affecting about 1 out of 1 million people per year worldwide. It usually affects individuals older than 60. CJD is one of the prion (PREE-awn) diseases. These diseases occur when protein, which is present throughout the brain, begins to assume an abnormal three-dimensional shape. This 
shape gradually triggers the protein throughout the brain to fold into the same abnormal shape, leading to increasing damage and destruction of brain cells. Recently, "variant Creutzfeldt-Jakob disease (VCJD)" was identified as the human disorder believed to be caused by eating meat from cattle affected by "mad cow disease." It tends to occur in much younger individuals, in some cases as early as their teens $[\mathbf{1 , 2 0 ]}$

\subsection{Normal pressure hydrocephalus}

Normal Pressure hydrocephalus (NPH) is another rare disorder in which fluid surrounding the brain and spinal cord is unable to drain normally. The fluid builds up, enlarging the ventricles (fluid-filled chambers) inside the brain. As the chambers expand, they can compress and damage nearby tissue. The "normal pressure" refers to the fact that the spinal fluid pressure often, although not always, falls within the normal range on a spinal tap [1, 20].

\subsection{Huntington's disease}

Hungtington's disease is a rare fatal brain disorder caused by inherited changes in a single gene. These changes lead to destruction of nerve cells in certain brain regions. Scientists identified the gene in 1993. Anyone with a parent with Huntington's has a 50 percent chance of inheriting the gene, and everyone who inherits it will eventually develop the disorder. In about 1 to 3 percent of cases, no history of the disease can be found in other family members $[\mathbf{1 , 2 0 ]}$.

\subsection{Wernicke-Korsakoff}

Wernicke-Korsakoff syndrome is a two-stage brain disorder caused by a deficiency of thiamine (vitamin B-1). Thiamine helps brain cells produce energy from sugar. When levels of the vitamin are too low, cells are unable to generate enough energy to function properly. Wernicke encephalopathy is the first, acute phase and Korsakoff psychosis is the long-lasting, chronic stage. The most common cause is alcoholism, but the syndrome can also be associated with AIDS, cancers that have spread through the body, very high levels of thyroid hormone, and certain other conditions $[\mathbf{1 , 2 0}]$.

Neural-Fuzzy is a fusion of neural network and fuzzy logic. Neuro-fuzzy system combines the advantages of both whilst eliminating their disadvantages. Using neuro-fuzzy logic the diagnosis of Alzheimer will provide a self-learning intelligent system that is capable of handling uncertainties in the diagnosis process.

\section{Literature Review}

Neural network $(\mathrm{NN})$ consists of an interconnected group of neurons [21]. Artificial Neural Network (ANN) is made up of interconnecting artificial neurons (Programming constructs that mimic the properties of biological neurons). A Neural Network is an analog and parallel computing system. A neural network is made up of a number of very simple processing elements that communicate through a rich set of interconnections with variable weights or strength. ANN 
(subsequently referred to as $\mathrm{NN}$ ) is used in solving artificial intelligence problems without creating a model of a real biological system. NN processes information using connectionist approach to computation. It changes it structures based on internal or external information that flows through the network during the learning phase. NN can be used to model complex relationship between input and output or find patterns in data. The term network in the term "Artificial Neural Network" arises because the function $f(x)$ is defined as a composition of other function $\mathrm{g}_{\mathrm{i}}(\mathrm{x})$ which can further be defined as a composition of the other functions [12]. Figure 1 present a simple NN which comprises of three layers (Input, Hidden and Output layers).

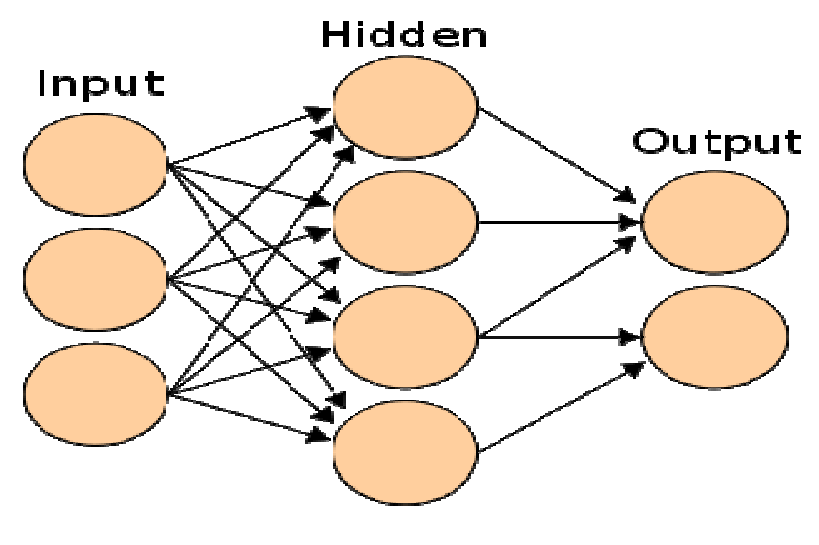

\section{Figure1: A simple Neural Network}

The NN presented in Figure 1, comprises of a layer of "input" connected to a layer of "hidden" units, which is in turn connected to a layer of "output" units. The activity of the input unit represented the raw information that is fed into the network; the activity of the hidden units is determined by the activity of the input unit and the weights between the hidden and output units. The hidden units are free to construct their own representation of the input; the weights between the input and hidden units determine when each hidden unit is active, and so by modifying these weights, a hidden unit can choose what it represents [8].

NN employs learning paradigm that includes supervised, unsupervised and reinforcement learning [27 and 28]. NN has been applied in stock market prediction, credit assignment, monitoring the condition of machinery and medical diagnosis [19, 24, 7, 28, and 3]. Application of $\mathrm{NN}$ in medical diagnosis includes electronic noses and diagonal of cardiovascular systems [29]. NN are ideal in recognizing diseases using scans. They learn by example, hence details of how to recognize the disease is not needed. What is needed is set of examples that are representatives of all the variation of the disease. However, NN cannot handle linguistic information and also cannot manage imprecise or vague information [2].

Fuzzy Logic (FL) is a branch of machine intelligence (Artificial Intelligence) that helps computers paint vivid pictures of the uncertain world. Fuzzy sets were introduced by [33] as a means of representing and manipulating data that are not precise, but rather fuzzy. Fuzzy logic provides an inference morphology that helps appropriate human reasoning capabilities to be 
applied to knowledge-based systems. The theory of fuzzy logic provides a mathematical strength to capture the uncertainties associated with human cognitive processes, such as thinking and reasoning.

A fuzzy set A is called trapezoidal fuzzy number (Figure 1) with tolerance interval [a, b], left width $\alpha$ and right width $\beta$ if its membership function has the following form

$$
A(t)= \begin{cases}1-(a-t) / \alpha & \text { if } a-\alpha \leq t \leq a \\ 1 & \text { if } a \leq t \leq b \\ 1-(t-b) / \beta & \text { if } a \leq t \leq b+\beta \\ 0 & \text { otherwise }\end{cases}
$$

and we use the notation $A=(a, b, \alpha, \beta)$. It can easily be shown that

$[\mathrm{A}] \gamma=[\mathrm{a}-(1-\gamma) \alpha, \mathrm{b}+(1-\gamma) \beta], \mathrm{V} \gamma \varepsilon[0,1]$.

The support of $A$ is $(a-\alpha, b+\beta)$.

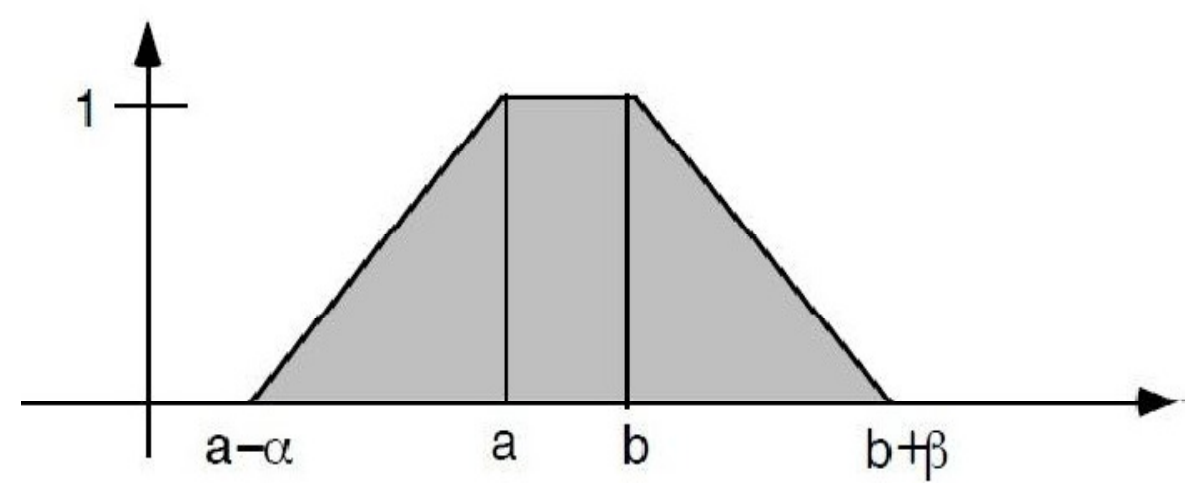

Figure 2: Trapezoidal fuzzy number

Fuzzy systems often learn their rules from experts. When no expert gives the rules, adaptive fuzzy systems learns by observing how people regulate real systems [15]. The difference between classical and fuzzy logic is something called "the law of excluded middle" [5]. In standard set theory, an object does or does not belong to a set. There is no middle ground. In such bivalent systems, an object cannot belong to both its set and its compliment set or to neither of them. This principle preserves the structure of the logic and avoids the contradiction of object that both is and is not a thing at the same time [31]. However, fuzzy logic is highly abstract and employs heuristic (experiment) requiring human experts to discover rules about data relationship. 
Fuzzy Neural Network or Neuro-Fuzzy system is a learning machine that finds the parameters of a fuzzy system (i.e., fuzzy sets, fuzzy rules) by exploiting approximation techniques from neural networks [23]. Neuro-fuzzy refers to the combination of artificial neural network and fuzzy logic. It eliminates the individual weaknesses of neural network and fuzzy logic while making use of their best advantages. Fusion of neural network and fuzzy logic (that is Neuro-fuzzy) is interesting [14, 13, 11, 17, 30, and 25]. Neuro-fuzzy system for the diagnosis of Alzheimer disease will provide a self-learning and adaptive system that is able to handle uncertain and imprecise data.

\section{Methodology}

The process for the medical diagnosis of Alzheimer starts when an individual consults a physician (doctor) and presents a set of complaints (symptoms). The physician then requests further information from the patient or from others close to him who knows about the patient's symptoms in severe cases. Data collected include patient's previous state of health, living condition and other medical conditions. A physical examination of the patient condition is conducted and in most cases, a medical observation along with medical test(s) is carried out on the patient prior to medical treatment.

From the symptoms presented by the patient, the physician narrows down the possibilities of the illness that corresponds to the apparent symptoms and make a list of the conditions that could account for what is wrong with the patient. These are usually ranked in the order (Low, Moderate and high). The physician then conducts a physical examination of the patient, studies his or her medical records and ask further questions, as he goes in an effort to rule out as many of the potential conditions as possible. When the list has been narrowed down to a single condition, it is called differential diagnosis and provides the basis for a hypothesis of what is ailing the patient. Until the physician is certain of the condition present; further medical test are performed or schedule such as medical imaging, scan, X-rays in part to conform or disprove the diagnosis or to update the patient medical history. Other Physicians, specialist and expert in the field may be consulted (sought) for further advices.

Despite all these complexities, most patient consultations are relatively brief because many diseases are obvious or the physician's experience may enable him to recognize the condition quickly. Upon the completion of the diagnosis by the physician, a treatment plan is proposed, which includes therapy and follow-up (further meeting and test to monitor the ailment and progress of the treatment if needed). Review of diagnosis may be conducted again if there is failure of the patient to respond to treatment that would normally work. The procedure of diagnosing a patient suffering from Alzheimer is synonymous to the general approach to medical diagnosis. The physician may carry out a precise diagnosis, which requires a complete physical evaluation to determine whether the patient have Alzheimer. The examining physician accounts for possibilities of having Alzheimer through an interview, physical examination and laboratory test. Many primary health care physicians may require tools for Alzheimer evaluation. 
A thorough diagnostic evaluation may include a complete history of the following:

a. When did the symptoms start?

b. How long have the symptoms lasted?

c. How severe are the symptoms?

d. Have the symptoms occurred before, and if so, were they treated and what treatment was received?

Neuro-fuzzy inference procedure is applied to the diagnosis of Alzheimer using the model prescribed in Figure 3. The decision support system using the neuro-fuzzy model is developed in an environment characterized by Microsoft Window XP Professional operating system, Microsoft Access Database Management system, Visual Basic Application Language and Microsoft Excel. Neuro-Solution and Crystal Report were used for Neural Networks analysis and graphical representation respectively.

\section{Result and Discussion}

To design our neuro-fuzzy system for diagnosis of Alzheimer, we designed a system which consists of a set of symptoms needed for the diagnosis (here, we are using nine basic and major symptoms):
a. Memory loss that affects day-to-day function
b. Difficulty performing familiar tasks
c. Problems with language
d. Disorientation of time and place
e. Poor or decreased judgment
f. Problems with abstract thinking
g. Misplacing things
h. Changes in mood and behavior
i. Changes in personality and Loss of initiative

Figure 3 presents the model of the Neuro-fuzzy system for the diagnosis of Alzheimer 


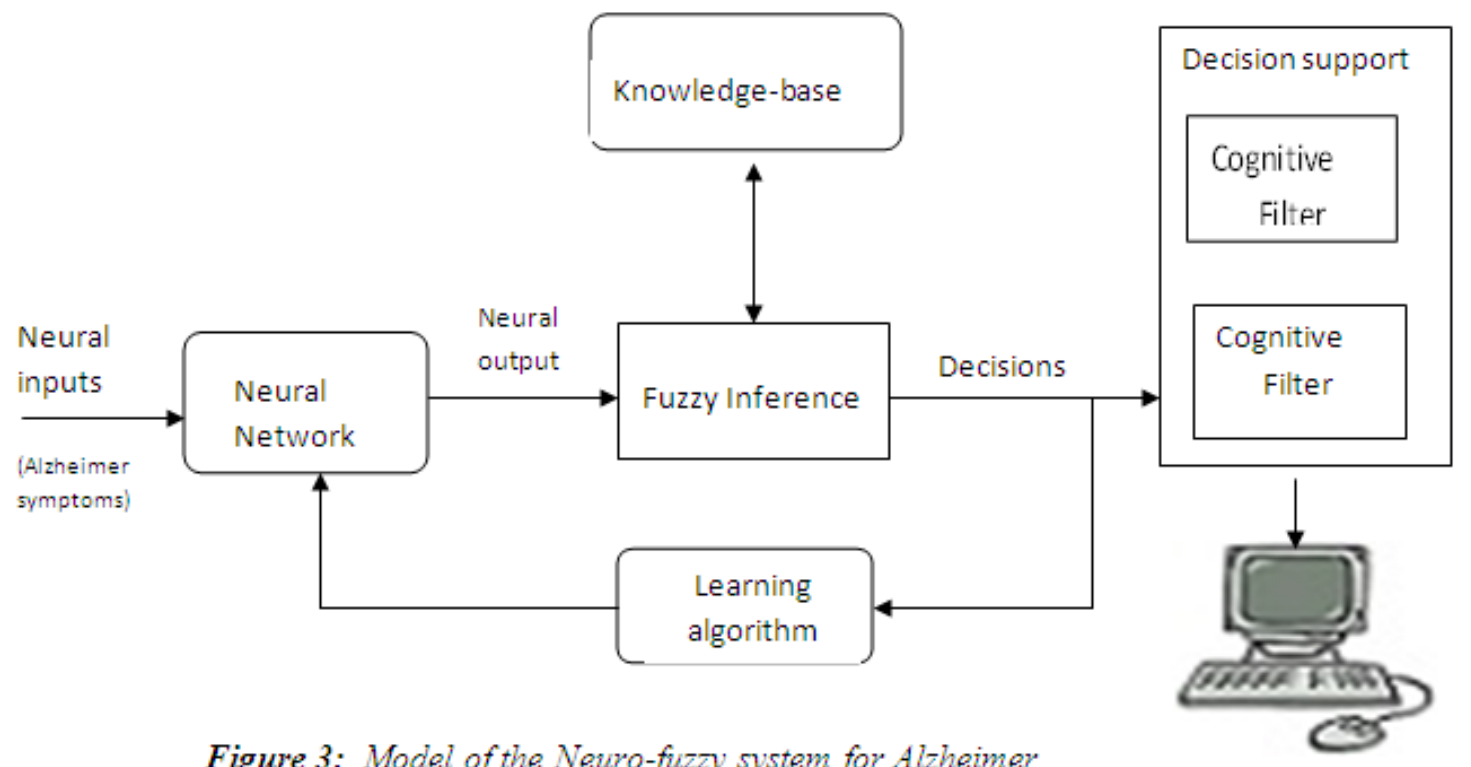

Figure 3: Model of the Neuro-fuzzy system for Alzheimer

The knowledge base consists of the database, which consist of nine basic parameters mentioned earlier. The values of the parameters are often vague (fuzzy) and imprecise hence the adoption of fuzzy logic in the model as means of analyzing these data. These parameters therefore constitute the fuzzy parameter of the knowledge base. The fuzzy set of parameters is represented by ' $\mathrm{P}$ ', which is defined as

$$
\mathrm{P}=\left\{\mathrm{P}_{1}, \mathrm{P}_{2} \ldots, \mathrm{P}_{\mathrm{n}}\right\}
$$

where $P_{i}$ represents the $j^{\text {th }}$ parameter and $n$ is the number of parameter (in this case $n=14$ ). The set of linguistic values which is modeled as a linker scale denoted by ' $\mathrm{L}$ ' is given as

$$
\mathrm{L}=\{\text { Low, Moderate, High }\}
$$

Neural networks provide the structure for the parameters, which serves as a platform for the inference engine. The inference engine consists of reasoning algorithm driven by production rules. These production rules are evaluated by using the forward chaining approach of reasoning [17]. The inference mechanism is fuzzy logic driven. The cognitive filter of the decision support engine takes as input the output report of the inference engine and applies the objective rules to rank the individual on the presence or absence of Alzheimer disease. The emotional filter takes as input the output report of the cognitive filter and applies the subjective rules in the domain of studies in order to rank individuals on the extent of the Alzheimer disease.

A universal set of symptoms of Alzheimer disease is set up for diagnosis where the patient is expected to choose or pick from the set of symptoms fed into the system. We used a simple 
binary encoding scheme wherein the presence of a symptom is represented by 1 in the input vector and 0 otherwise (we call this the symptom vector).

The operational procedure of the model is represented in Figure 4. The set of symptoms are fed into the network. The patient is expected to choose from the list of symptoms the one corresponding to what he/she is having

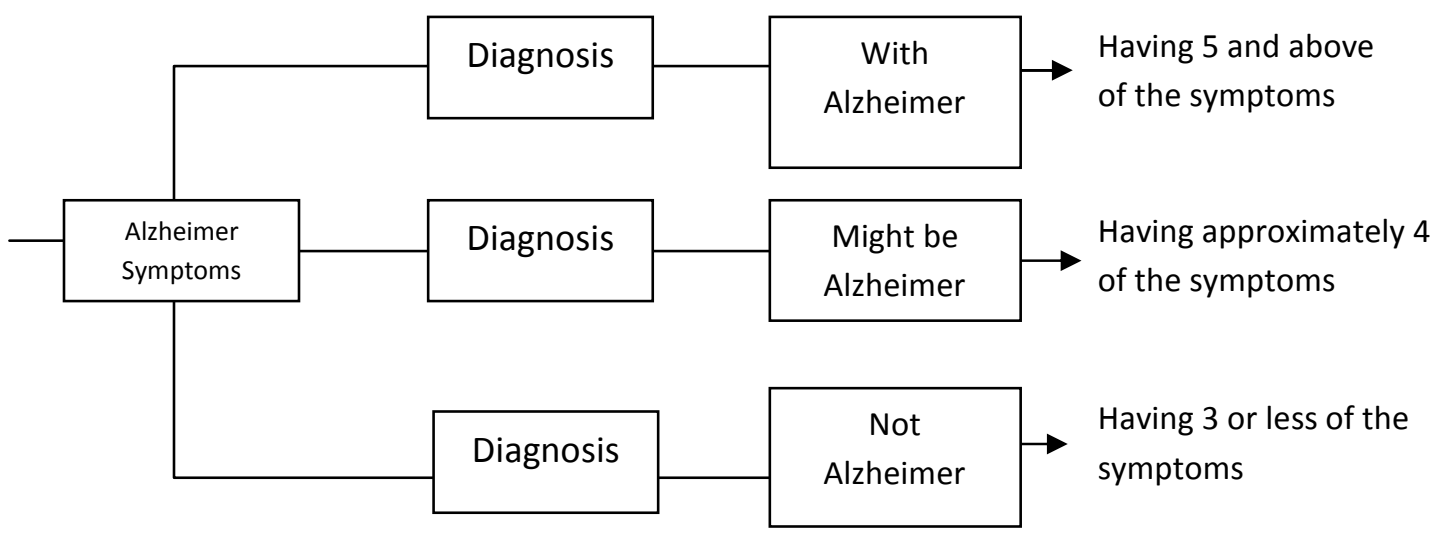

Figure 4: Operational Procedure of the Neuro-Fuzzy system for the diagnosis of

\section{Alzheimer}

If the patient is having five or more of the symptoms, he is having severe Alzheimer and should go for treatment urgently. If it is approximately four of the symptoms he is having, he might be suffering from Alzheimer and hence should see a physician right away, but if it is three or lesser of the symptoms, he may not be having Alzheimer.

A typical that contains the nine symptoms of Alzheimer presented in Table 1. This shows the degree of intensity of the symptoms. As the value tends to 1.0, the more the chances that the patient is suffering from Alzheimer.

Table 1: Data Set showing the degree of intensity of Alzheimer Symptoms

\begin{tabular}{|l|c|c|c|}
\hline SYMPTOMS & \multicolumn{3}{|c|}{$\begin{array}{c}\text { DEGREE OF INTENSITY } \\
\text { Scale (0.00 - 1.00) }\end{array}$} \\
\hline $\begin{array}{l}\text { Memory loss that affects day-to-day } \\
\text { function }\end{array}$ & 0.60 & 0.30 & 0.10 \\
\hline Difficulty performing familiar tasks & 0.30 & 0.55 & 0.15 \\
\hline Problems with language & 0.80 & 0.10 & 0.10 \\
\hline Disorientation of time and place & 0.68 & 0.15 & 0.17 \\
\hline Poor or decreased judgment & 0.32 & 0.60 & 0.08 \\
\hline
\end{tabular}


International Journal on Soft Computing ( IJSC ), Vol.2, No.2, May 2011

\begin{tabular}{|l|c|c|c|}
\hline Problems with abstract thinking & 0.59 & 0.29 & 0.12 \\
\hline Misplacing things & 0.20 & 0.15 & 0.65 \\
\hline Changes in mood and behavior & 0.18 & 0.70 & 0.12 \\
\hline $\begin{array}{l}\text { Changes in personality and Loss of } \\
\text { initiative }\end{array}$ & 0.50 & 0.50 & 0.00 \\
\hline RESULT & $\begin{array}{c}\text { With } \\
\text { Alzheimer }\end{array}$ & $\begin{array}{c}\text { Might be } \\
\text { Alzheimer }\end{array}$ & $\begin{array}{c}\text { Not } \\
\text { Alzheimer }\end{array}$ \\
\hline
\end{tabular}

Next, we create fuzzy logic membership functions that define the value of input/ output terms used in the rules. Membership functions are graphical function representation of the magnitude of the preparation of each input that is processed. Typical membership function is presented in Figure 5. Figure 6 shows that the height of the symptoms is $0.0,0.5$ or 1.0 and does not exceed 1.0. The fuzzy set however is zero, X/4 or one. From Figure 6, we say that when the fuzzy set is between zero and X/4, the person's condition is Low ("Not suffering from Alzheimer"). When the fuzzy set is in-between zero and one, the condition is moderate ("might be suffering from Alzheimer") and when it is between X/4 and one, the person's condition is high ("suffering from severe Alzheimer").

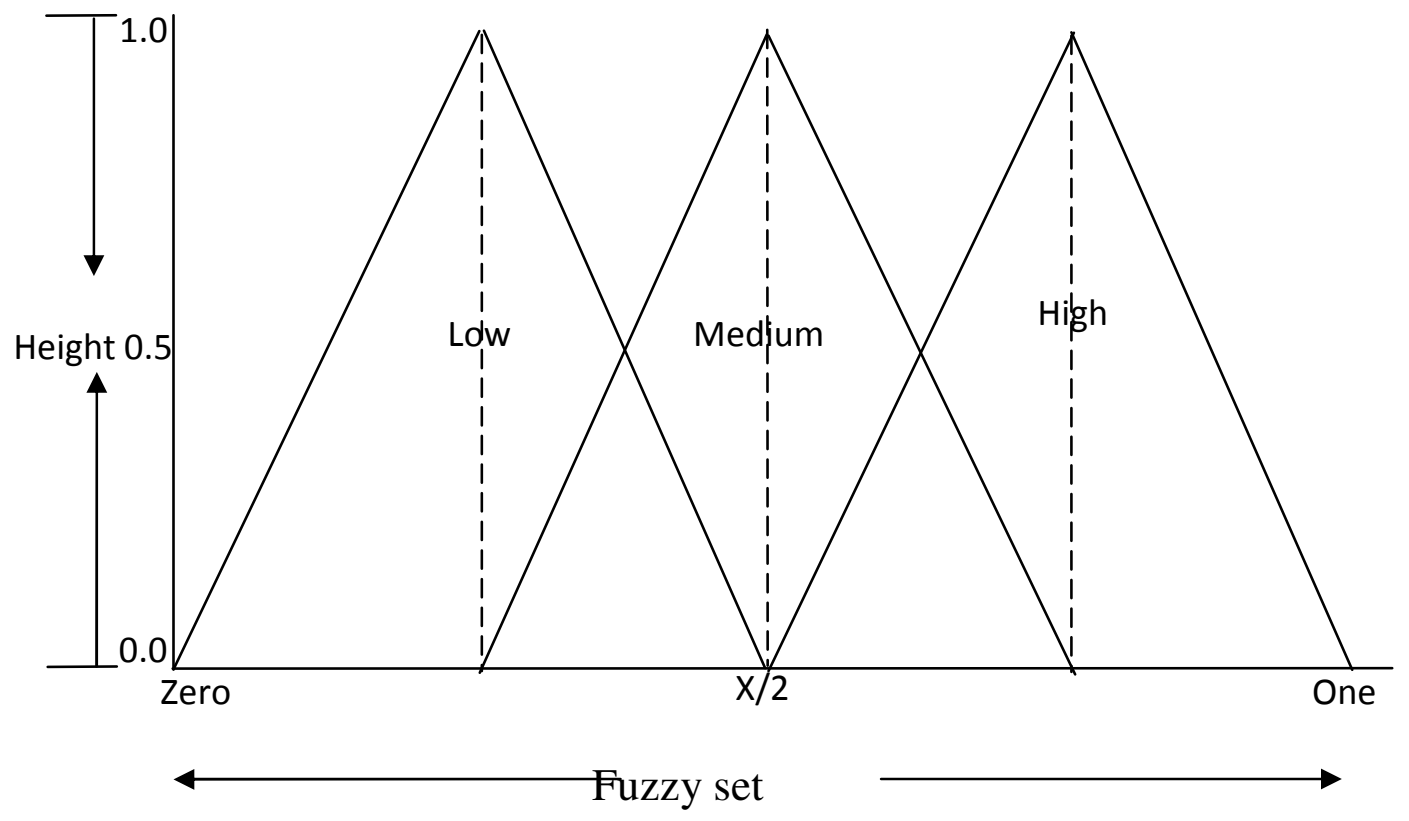

Figure 5: Membership Function for Alzheimer

Further, we create the necessary pre and post processing. As inputs are received by the system, the rule based is evaluated. The antecedent, which is the (IF X AND Y), block test the input and produces a conclusion. The consequent (THEN Z) are satisfied while the others may not be. The 
conclusion is combined to form logical sums. The degree of membership (D.O.M) of Alzheimer is represented in Figure 6

\section{D.O.M}

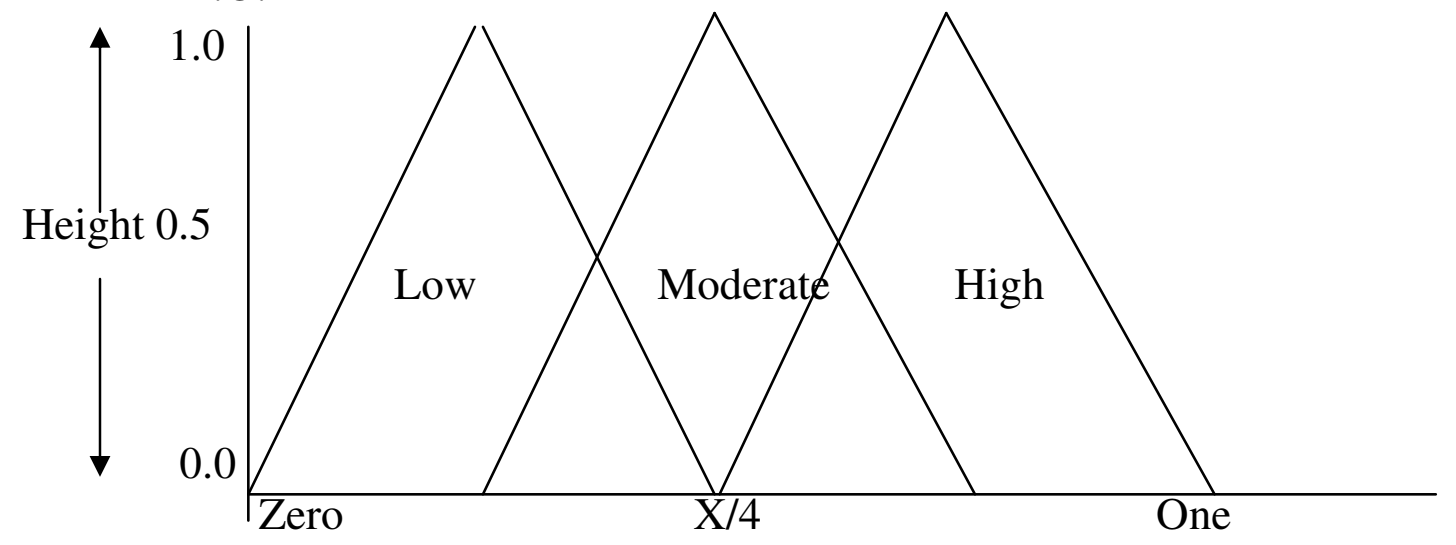

Alzheimer

\section{Symptoms}

\section{Figure 8: Analysis of Alzheimer}

Defuzzification coverts the rules base fuzzy output into non-fuzzy (numerical values). It reflects the interpretation of the logic of the different linguistic variable. The system can also be configured to handle not only Alzheimer but, other kind of illness and diseases.

\section{Conclusion}

The need to design a system that would assist physician in medical diagnosis of Alzheimer cannot over be emphasized. This paper which demonstrates the practical application of Information Technology (IT) in the health sector, presented a hybrid neuro-fuzzy system to help in diagnosis of Alzheimer using a set of symptoms. This system which uses a set of fuzzified data set incorporated into neural network system is more precise than the traditional system. The system designed is an interactive system that tells the patient his current condition as regards Alzheimer. It should however be noted that the system was not designed to give prescription of Alzheimer drugs to patients but can also be expanded to do so in subsequent research. A system of this nature that has the ability to diagnose a person suffering from Alzheimer should be introduced in health care delivery centers and hospitals to help ease the work of physicians. 


\section{References}

[1]. Alzheimier Association (2010), "What is Alzheimer", retrieved from http://www.alz.org/alzheimers_disease_what_is_alzheimers.asp

[2]. Akinyokun O.C. (2002), "Neuro-fuzzy expert system for evaluation of human Resource performance", First Bank of Nigeria Endowment Fund lecture Federal University of technology, Akure, Nigeria.

[3].Aleksander I. and Morton H. (1998), "An introduction to neural computing" $2^{\text {nd }}$ Edition Computer Science press.

[4]. Andreas N. (2001), "Neuro-Fuzzy system", retrieved from http//:Neuro-Fuzzy System.html.

[5].Bart K. and Satoru I. (1993), "Fuzzy Logic", retrieved from http//:Fortunecity.com/emachines/e11/86/fuzzylog.html.

[6].Beth R. (2002), "Alzheimer's Disease: A Brief History and Avenues for Current Research", retrieved from http ://www.jyi.org/volumes/volume6/issue2/features/reger.html -Cached Similar

[7]. Bishop C.M. (1995), "Neural Networks for pattern Recognition", Oxford University Press, United Kingdom.

[8].Christos S. and Dimitros S. (2008), "Neural Network" retrieved from http//:docs.toc.com/doc/1505/neural-networks.

[9].CWS.(2000), “Tuberculosis Research Paper”, $\quad$ retrieved from http://writing4students.blogspot.com/2009/12/tuberculosis-research-paper.html.

[10]. Edward C.H. (2010), "Article: The gorilla Connection" retrieved from http//:Nature.com/nature/journal/v467/n7314/full/467404a.html.

[11]. Eklund D. and Fuller R. (1993), "A Neural-Fuzzy Approach to medical Diagnostic" Gedemedic project, Abo Academy University, Development Centres heisnki, pp.210-225.

[12]. Gary R. and George P.E. (2002), "Application of Neuro System to behavior Representation in Computer generated forces", retrieved http//:Cuil.com.

[13]. Johnson R.C. (1993), "Making the Neural-Fuzzy Connection", Electronic Engineering Times, Cmp Publications, Manhasset, New York.

[14]. Kosaka M. (1991), "Application of Fuzzy Logic/Neural Network to Securities Trading Decision Support", Conference Proceeding of the 1991 IEEE International Conference on Systems, man and Cybernetics, Vol.3, pp.1913 - 1918.

[15]. Leondes C. (2010), "The Technology of Fuzzy Logic Algorithm retrieved From Suite101.com/examples-of-expert-System-application-in-artificial Intelligience.

[16]. MedicineNet (2011), “Alzheimer" retrieved from http://www.medicinenet.com/script/main/art.asp?articlekey=505\&page=4

[17]. Nauck K. (1996), "Fuzzy Neural Network", http//:Wikipedia.org.

[18]. Neil and Janet C. (2005), "History of Tuberculosis", retrieved from http:// www.micklebring.com/Oakwood/ch18.htm

[19]. Pao Y.H. (1989), “Adaptive Pattern Recognition and Neural Network”, Addison Wesley. 
[20]. Peter V. R.; Deborah B.; Berry W.R.; Teresa R.; Lon S.S.Pierre N.T., David M.B. (2011), "Practice guideline for the treatment of patient with Alzheimer's and other Dementia" $\begin{array}{ll}\text { retrieved from } & \end{array}$ http://www.psychiatryonline.com/pracGuide/PracticePDFs/AlzPG101007.pdf

[21]. Ponniyin S.K (2009), "Neural Network”, Icann2007.org/neural.networks.

[22]. Otuorimuo O. (2006), "Prototype of Fuzzy System for the Formulation and Classification of Poultry Feed", Bachelor of Science (Computer Science) Project, University of Benin, Benin City, Nigeria.

[23]. Rudolf K. (2008), “Article: Institute of Information and Communication System”, OttoVan-Guericke, University of Magdebury, Germany.

[24]. Rumelhert D.E.,Windrow B., and Lehr M.A (1994), "Neural Networks: Application in Industry, Business and Science", Communication of ACM,37(1994), 93-105.

[25]. Stathacopoulou R.,Magoulas G.D.,Grigoriadou M., and Samarakou M.(2004), "NeuralFuzzy knowledge processing in Intelligent learning Environment for Improved Student Diagnosis" DOI Information 10.1016/j.ins.2004.02.026.

[26]. Statsoft Incorporated (2008), "Neural Network" retrieved from http//:google.com.

[27]. Tom S.; Justin B.; Daan W.; Yi Sun; Martin F.; Frank S.; Thomas R and Jurgen S(2010), "PyBrain" Journal of Machine Learning Research 1 (2010) 999-1000 retrieved from http://www.idsia.ch/ sun/pybrain.pdf

[28]. Vahid K. and Gholam A.M. (2009), "Artificial Intelligence in medicines”,V47 , Issues 1 Information Technology Department, School of Engineering, Terbiat Moderas University Tehran,Iran.

[29]. Wikipedia (2010), “Artificial Neural Network" retrieved from http//: en.Wikipedia.org/wiki/Artificial-neural-network.

[30]. Wong K., Fung C and Myers D. (2002), "An Integrated Neural Fuzzy Approach With reduced rules for well log analysis”, International Journal of Fuzzy Systems 4(1) 592-599.

[31]. World Health Organization (2005), "International Publication: Malaria", retrieved from Whqlidboc.Who.Int/Publication/2005/9241580364-Chapter 7.pdf.

[32]. WrongDiagnosis, (2011), "Alzheimer: diagnosis and prognosis", retrieved from http:// wrongdiagnosis.com

[33]. Zadeh L.A. (1965), "Fuzzy sets. Information and control, Vol.8, pp.338-353.

[34]. Zimmermann H.J. (1993), "Fuzzy sets, Decision making and expert system" International series in Management Science/Operation Research, University of Houston, U.S.A. 